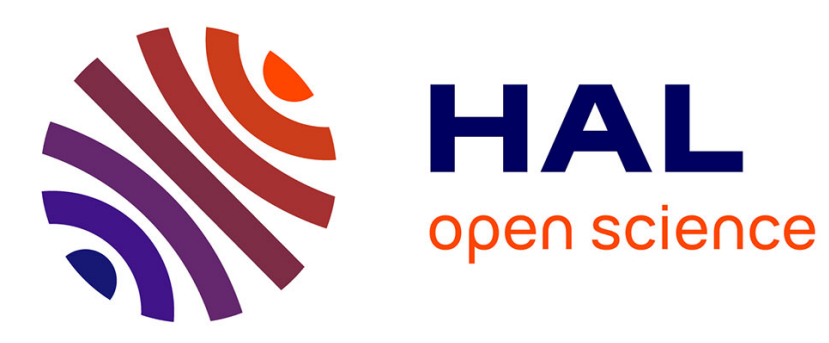

\title{
Parametric Continuous Curvature Path for Smooth Steering with the Car-like Vehicles
}

Suhyeon Gim, Lounis Adouane, Sukhan Lee, Jean-Pierre Derutin

\section{To cite this version:}

Suhyeon Gim, Lounis Adouane, Sukhan Lee, Jean-Pierre Derutin. Parametric Continuous Curvature Path for Smooth Steering with the Car-like Vehicles. 13th International Conference on Intelligent Autonomous System (IAS-13), Jul 2014, Padova, Italy. hal-01712934

\section{HAL Id: hal-01712934 \\ https://hal.science/hal-01712934}

Submitted on 23 Feb 2018

HAL is a multi-disciplinary open access archive for the deposit and dissemination of scientific research documents, whether they are published or not. The documents may come from teaching and research institutions in France or abroad, or from public or private research centers.
L'archive ouverte pluridisciplinaire HAL, est destinée au dépôt et à la diffusion de documents scientifiques de niveau recherche, publiés ou non, émanant des établissements d'enseignement et de recherche français ou étrangers, des laboratoires publics ou privés. 


\title{
Parametric Continuous Curvature Path for Smooth Steering with Car-like Vehicles
}

\author{
Suhyeon Gim ${ }^{1,2}$, Lounis Adouane ${ }^{1}$, Sukhan Lee $^{2}$, and Jean-Pierre Derutin ${ }^{1}$ \\ 1 Institut Pascal/Université Blaise Pascal, 63177, Aubiére Cedex, France \\ 2 SungKyunKwan University, Seoburo 2066, 440-746, Suwon, Korea
}

\begin{abstract}
This paper proposes a solution to obtain parametric continuous curvature path for nonholonomic car-like vehicle and addresses also efficient algorithm to obtain an appropriate reference path for smooth path following. As a local planner using parametrically adjustable clothoid, the proposed solution, of constant sharpness, permits to obtain continuous curvature and thus smooth steering behavior of the vehicle which enhances the passenger comfort. The proposed parametric continuous curvature path is integrated in a global planner which includes an appropriate way of isolating the boundary conditions of each local planner. This last characteristic is useful to reconstruct a smooth reference path from raw data of actual vehicle trajectory. The solution is applied to a real road datasets and tested using Lyapunov based controller for validating the effectiveness on path following performance. The simulated results show reliable and enhanced performance for vehicle path following when the proposed algorithms are used.
\end{abstract}

\section{Introduction}

Autonomous navigated vehicle has attracted a lot of researchers during more than a decade. Its fundamental technologies on commercial usage are also growing with the innovative computational and systematic performance. The path generation or trajectory generation in the autonomous navigated vehicles is the core technology which is still under development and much attentions are paid to its practical implementation for assuring the passenger comfort with safe performance. The path generation for the nonholonomic car-like vehicle makes the path following be harder and difficult to reorient due to its nonholonomic characteristic with kinematic and dynamic constraints. There have been a lot of smooth path generation methods for the vehicle and the continuous curvature path has been focused for its close relationship with vehicle parameters and driving comfort $[1,2]$ The smooth path for the nonholonomic vehicle have been investigated by numerous groups. Dubins [3], Reeds-Shepp [4] and Fraichard-Scheure [5] are prominent pioneering works and its advanced model are carried out by other groups such as $[6,7,8,9]$. The path is composed by clothoids, straight lines and circular arcs segments for its continuity of curvature under the parametrical constraints. [5] solved the continuous curvature steering problem for car-like vehicle with constraints of upper bounded curvature and upper bounded curvature 
rate (or sharpness) which extends the Reeds and Shepp's path [4]. The authors in [10] proposed an infinitely differentiable smooth paths which approximate Dubin paths with bounded curvature and sharpness. The work proposed in [1] tries to solve the overtaking problem in the road based on clothoidal trajectories by approximating the rational Bézier curves, it used the homotetical factor, adjusted by scalable parameter and control points, however the solution needs a large amount of calculation for multi dimensional linear algebraic equation to find the Bézier control points. It is also difficult to acquire the curvature information from the resultant curve. [11] addresses the clothoidal segments problems in order to satisfy the two already defined end configurations using rigorous mathematical analysis and while proving the solvability and solution uniqueness. However, the proposed solution and its algorithm lack on the curvature and sharpness parameters analysis and thus, it is hard to get the curvature diagram from the solution. Moreover, a paired clothoids are managed by control points which should be pointed manually by human designer. One of the most noticeable works to be referenced is [12]. It presented a simple and fast trajectory generation method of continuous curvature with minimum sharpness as human natural and safe driving. However, the solution was limited only for the lane change maneuver example. For the path following issues, [13] produces a smooth trajectory in the respect to the standard human comfort while following the trajectory using sliding mode control. The trajectory comes from the predefined quintic polynomial but, the presented results have different steering angle discontinuous. It could be expected that the system may suffer from other errors in the real vehicle experiments linked to these discontinuouty in the steering set-points. Authors in [2] propose smooth trajectory using cubic and trigonometric splines. The obtained control results were compared to a clothoidal planning while considering human comfort criteria. It concluded with the remark that the use of the clothoid curves is better for the vehicle performances but it is much more complex and harder to obtain these clothoids (mainly when the modification of the trajectory occurs). In [14], automatic multi-vehicle navigation for urban transportation is proposed. Vehicles shares a common reference trajectory with the compensation of the localization by outward sensors. The used trajectories was locally optimized by $B$-spline interpolation method. Artificial Potential Field methods [15] are among the most interesting and really broadcasted work in the literature for path planning. Authors in [16] used this technic for planning and re-planning one mission for a group of mobile robots which evolve in structured environment to attain different way-points. Even if the obtained results gave an interesting tool for making a decision (which trajectory to take by each robot) in complex context as the multi-robot and multi-target mission, the obtained trajectories did not take into account the different robots' structural constraints and thus the planned missions can not be reached, in certain configurations. This paper proposes a solution of Parametric Continuous Curvature Path (pCCP) using algorithm which adjusts the clothoid's parameters. The proposed methodology is applied to the well-defined local boundary conditions [5, 17] and then implemented for an effective path following problem. The proposed solution aims at 
reconstructing one reference path, as close as possible from the original path, permitting thus the smoothing and/or slightly replanning of the already predefined path with one which be close and with continuous curvature.

Therefore, the proposed $\mathbf{p C C P}$ algorithm could be useful for making a reference path or reconstructing one from an already obtained raw data in the global frame work $[8,13]$. To validate the reliability of the obtained paths, these path was followed by a robot while using Lyapunov based controller [18, 19]. The simulated results show reliable and better path following behavior using obtained smooth path. This paper is organized as following. In the next section, the path following model for the nonholonomic vehicle and the problem is defined. In section 3, the proposed clothoids solutions for the path generation are given with algorithmic description. In section 4 , the smoothing procedure for the application to a practical implementation is addressed. It shows some simulated results. Section 5 gives finally some conclusions and prospects.

\section{Path following model for nonholonomic car-like vehicle}

\subsection{Kinematics modeling for path following}

Nonholonomic car-like vehicle is driven by acceleration/brake mechanism at rear wheels while it is steered by steering actuator at front wheels. The vehicle has a nonholonomic kinematics model where it is assumed that there exists no rolling contact-slip on the ground surface The steering angle corresponds to the curvature of the robot (cf. Fig. 1).When the vehicle is modeled as a point of the motion center, the nonholonomic vehicle kinematics are represented by following differential form.

$$
\begin{aligned}
\dot{x}(t) & =v(t) \cdot \cos \theta(t) \\
\dot{y}(t) & =v(t) \cdot \sin \theta(t) \\
\dot{\theta}(t) & =\frac{v(t)}{L} \cdot \tan \gamma(t)
\end{aligned}
$$

where, $v(t)$ and $\theta(t)$ are the linear driving velocity and orientation angle at time $t$ respectively and $x(t), y(t)$ middle position of rear wheels (where $x$ and $y \in \mathbb{R}^{2}$ ) represent the position of the vehicle. $\gamma(t)$ is the steering angle of the vehicle at time $t$ and it is related to the curvature $\kappa(t)$ where the radius of curvature $\rho(t)$ is defined by the two axis interconnection at the Instantaneous Center of Rotation (ICR). $L$ is the distance between frontal and rear axis. For the description of the whole path, above equations requires the whole information on the velocity and steering angle through the time. Different to the trajectory tracking, the path following requires for the vehicle to converge and follow the path without time specification. The major control is performed thus by its steering wheels (cf. Fig. 1) with generally constant forward velocity. For smoother convergence to the desired path, virtual target projected of the actual vehicle on the path is tackled to bypass some problem of the feedback controller [20] where the target 
is defined on Frenet-Serret frame $\mathcal{F}$. Fig. 1 depicts the nonholonomic car-like vehicle based on virtual target for the path following on the global coordinate $X-\mathrm{Y}$.

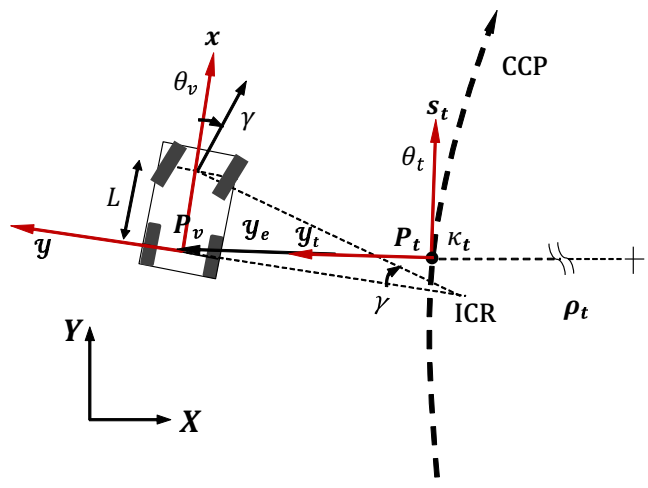

Fig. 1. Path following model for the car-like vehicle

In Fig. 1, the local frame of the vehicle is located on its motion center, $P_{v}$ with orientation $\theta_{v}$ and its virtual target frame places on $P_{t}$ with $\theta_{t}$. At $P_{t}$ on the path, the pose is described by curvilinear abscissa $s_{t}$ and it has curvature $\kappa_{t}$ and radius of curvature $\rho_{t}$. From above definition, following equations presents the kinematic relations on the Frenet frame $\mathcal{F}$.

$$
\left\{\begin{array}{l}
\dot{s}_{e}=v \frac{\cos \theta_{e}}{1-y_{e} \kappa\left(s_{e}\right)} \\
\dot{y}_{e}=v \sin \theta_{e} \\
\dot{\theta}_{e}=v\left(\frac{\tan \gamma}{L}-\frac{\kappa\left(s_{e}\right) \cos \theta_{e}}{1-y_{e} \kappa\left(s_{e}\right)}\right) .
\end{array}\right.
$$

In $(4), \mathcal{F}$ is represented as $\left(s_{t}, y_{t}\right)$ at $P_{t}$ and $\theta_{e}=\theta_{v}-\theta_{t}$ where $\theta_{v}$ and $\theta_{t}$ is measured counterclockwise from $\mathrm{X}$-axis on the global coordinate. The control strategy for path following must permit to have $y_{e}, \theta_{e}$ to converge to 0 while keeping $1-y_{e} \kappa\left(s_{e}\right) \neq 0$. For the strategy of path following based on virtual target, Lyapunov based controller are augmented to extend kinematic control to a dynamic consideration as well as its robustness to the parametrical modelling uncertainties [21].

\subsection{Clothoids representation with basic properties}

There is a useful mathematical representation for the nonholonomic vehicle path according to its length (curvilinear abscissa) where it uses clothoid. A clothoid is represented by curvature variation with the length. Fully defined clothoidal form for a path is efficient for car-like vehicle in that it gives information on the curvature along the length and it could also give maneuvering 
information to the vehicle driver of the vehicle located on its local or moving coordinate frame (cf. Fig.1). By the definition of clothoid (or Euler spiral) [11], the coordinates are consecutively defined along the length $s$. A clothoid is defined by parametrical forms given by,

$$
\begin{gathered}
\kappa(s)=\kappa_{0}+\alpha s \\
\theta(s)=\int_{0}^{s} \kappa(u) d u \\
x(s)=\int_{0}^{s} \cos \theta(u) d u \\
y(s)=\int_{0}^{s} \sin \theta(u) d u
\end{gathered}
$$

where, $\alpha$ is the sharpness (or rate of curvature) for curvature $\kappa(s)$. Equation (5) shows that the curvature increase or decrease by constant sharpness $\alpha$ and the orientation in (6) changes with integration of curvature in (5). It is to be noted that this mathematical relation is same as the physical relation between the steering angle and the vehicle orientation where the orientation varies in integrated amount of steering changes. It is also noted that the position in coordinate is determined only after the orientation at a length is calculated as in (7), (8). This means that to find the sharpness for a clothoid, to meet the desired position and orientation at a certain curvilinear distance, requires to have at least an analytic model of the inverse kinematic solution. This model is unfortunately not available yet in the literature. In fact, the problem is very complex to resolve.

A clothoid has the property to have continuous curvature which is either increasing or decreasing through the length. There are some kinds of formulations for the rate of the curvature such as polynomials, exponential or trigonemetric functions with composition. However, the $1^{\text {st }}$ order form 5 , relying on the constant sharpness, is well-known not only for its computational simplicity but also for phenomenal similarity to the real vehicle actuation system. To be more specific, the curvature of a point on the path corresponds to the steering angle of the vehicle which follows the path at the point and the sharpness signifies the rate of the steering change at that point. Thus, the sharpness is related to the steering actuation which exerted by human handling motion or steering motor system.

To specify the convergence criteria as well as determining initial values for the algorithm, some basic properties or pattern according to the parameter variation are useful to understand. When a clothoid is defined, the relations among the parameters are presented as follows,

$$
\kappa=\sqrt{2 \delta \alpha}, \delta=\frac{\kappa^{2}}{2 \alpha}, s=\sqrt{\frac{2 \delta}{\alpha}} .
$$

where all parameters are the values at the end point of the clothoid and $\delta$ means the amount of orientation change through the whole length which is 
called deflection [2]. Note that $\alpha, \kappa, \delta$ and $s$ are closely related to each other and furthermore, if two of them are defined, then the others are also determined. This relation is important to make a clothoid meet a desired pose by adjusting independent two parameters, e.g. $(\alpha, \kappa)$ or $(\alpha, \delta)$. One can find some important properties for the clothoidal segment that the curvature, sharpness, length and deflection are closely related to each other. In the following analysis, some regular and scalable patterns for the parameters conditions could be found. In Fig. 2,

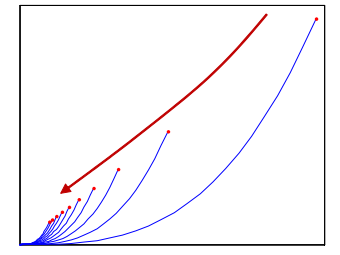

(a) $\alpha \uparrow, \delta *$

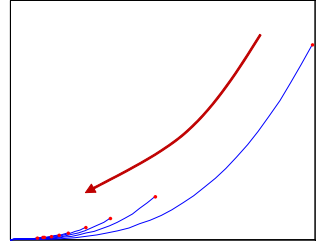

(b) $\alpha \uparrow, \kappa *$

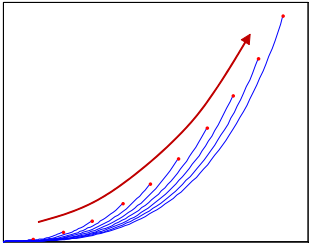

(c) $\delta \uparrow, \kappa *$

Fig. 2. Basic observed properties for parametrical variation on a clothoid

one parameter varies whereas the other stay constant which are denoted as $\uparrow$ and $*$ respectively. The long (red) arrow in Fig. 2 indicates the varying direction of the clothoid according to its parametrical change. From the observed results, one can find that the shape of the clothoid shrinks or expands as the sharpness $\alpha$ varies. In addition, the position at the clothoidal end moves on shrinking or expanding direction. It is also noticeable that while its shape and position moves, the other parameter imposes the end pose of the segment, i.e. the orientation or curvature. Such a pattern is used as an important property in the proposed algorithm (cf. section 3) to find the solution of the clothoid parameters.

\section{Continuous Curvature Path generation}

\subsection{Problem definition and its solution}

This subsection deals with the problem of generating a smooth path in local planner for a nonholonomic car-like vehicle. The local planner acts as generating a shorter trajectory or path for the minimum allowable distance in the configuration defined by boundary condition. The configuration $P$ is represented by four variables $x, y, \theta$ and $\kappa$ that determines the vehicle motion along the path, which correspond respectively to $x$ and $y$ vehicle position, orientation and the curvature at that position.

For pCCP generation, clothoid is the major component on the trajectory and each clothoid is composed with other segments satisfying pose configuration with attributed constraints. Well-defined path of continuous curvature guides 
the vehicle to follows the path with smoothing steering pattern. For pCCP as a local planner, it requirs at least two clothoids to satisfy the both end configurations where one clothoid increases from initially zero curvature and then the other decreases to final zero curvature. The first clothoid is by $C_{1}$ and the second by $C_{2}$ and their composition is defined by $C_{1} \oplus C_{2}$. This composition must satisfy the orientation continuity $G^{1}$ as well as curvature continuity $G^{2}$ where $G^{n}$ is $n$-th order of Geometric Continuity [22]. In this work, we make a focus on the path generation for a car-like vehicle in a road environment.

Problem: From $P_{i}\left(x_{i}, y_{i}, \theta_{i}, \kappa_{i}\right)$ to $P_{f}\left(x_{f}, y_{f}, \theta_{f}, \kappa_{f}\right)$ passing through a point $P_{c}$ at its maximal curvature $\kappa_{\max }$, with $\kappa_{i}=0$ and $\kappa_{f}=0$, find the minimum number of clothoids which satisfy the both configurations with curvature continuity along the path.

Proposition 1. If the both end poses could not be accessed by clothoidal parametric variation, straight line or circular arc segments are added with the conformity on the orientation $G^{1}$ and curvature continuity constraint $G^{2}$.

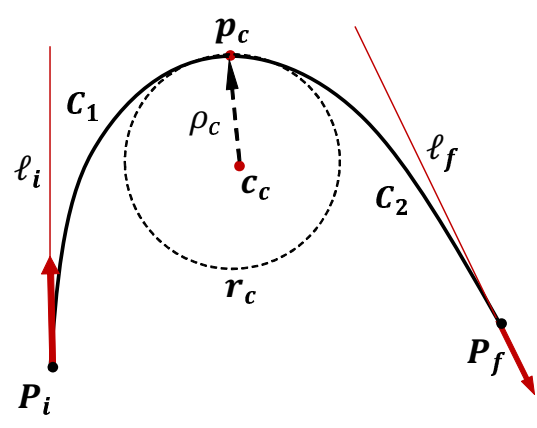

(a) Two clothoids composition

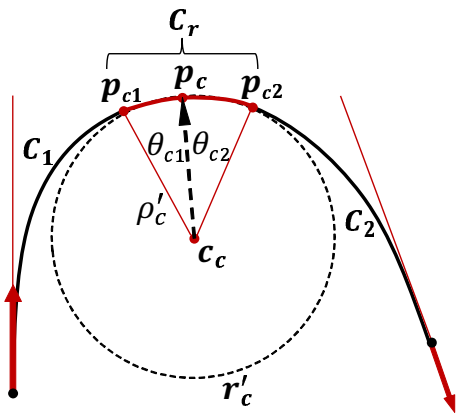

(b) Two clothoids and inter circular arc

Fig. 3. Basic pCCP problem

Fig. 3(a) depicts the defined problem. The initial pose $P_{i}$ and the final pose $P_{f}$ are denoted by red arrows and the extension lines are displayed from each pose as $\ell_{i}$ and $\ell_{f}$ respectively. While fulfilling the pose configuration at both ends, clothoid segment $C_{1}$ and $C_{2}\left(\forall s \in\left[s_{0}, s_{l}\right]\right)$ are generated from the point $p_{c}$ with same orientation and curvature to meet $\ell_{i}$ and $\ell_{f}$ at $P_{i}$ and $P_{f}$ respectively, where the inner-tangential circle $r_{c}$ with a radius $\rho_{c}$ and the center $c_{c}$. For above case of $\frac{\pi}{2}<\left|\theta_{i}-\theta_{f}\right|<\pi$, the minimal number of clothoid is two as proven in [11]. Note that $p_{c}$ is added to satisfy the solution. With the initial pose and final pose configuration defined in the problem, there are numerous solution to converge to $\ell_{i}$ or $\ell_{f}$. However, to make the reconstructed path closer to the original path 
(cf. Fig. 5(a)), additional constraint is required. In our problem, the objective is fulfilled by adding the maximal curvatured point $p_{c}\left(\kappa_{\max }\right)$. The position $p_{c}$ in the path gives an information on the vehicular steering behavior such that until this position, the steering rotate up to its maximal turn. Then it turns back to its middle center where former part is represented by the first clothoid and later part is expressed by the second clothoid. Another importance in fixing $p_{c}$ corresponds to when a vehicle path includes some region of constant angle of steering which is drawn as an circular arc segment. As usual, this steering behavior is shown at the region of highly curvatured road geometry section such as road intersection. In this case, the resultant solution only only two clothoids withot any circular arc segment could contain large differences with regard to the original path.

Fig. 3(b) shows the case where an circular arc segment is included between two clothoids. This solution is imposed in the cases where the both end positions are too close or/and the maximal curvature constraint is reached. Note that with the same configurations, the maximum curvature as given in Fig. 3(a) is greater then the case in (b) while it explicitly true if $\rho_{c}<\rho_{c}^{\prime}$. This case is mainly useful for reconstructing the origianl reference path having regions of constant curvature as dealt with in the demonstrative example given in 3.

The proposed methodology to resolve the presented problem is firstly to determine $p_{c}$ with $\ell_{i}$ and $\ell_{f}$ from $P_{i}$ and $P_{f}$. The determination of $p_{c}$ is described in the procedure given in Algorithm 2. By adjusting $\alpha_{1}$ and $\delta_{1}, C_{1}$ is generated until its end point $C_{1}\left(s_{0}\right)$ to meet $\ell_{i}$ for the distance to converge within a small constant threshold value defined by designer as $D_{1}^{e}<\varepsilon$ ( $\varepsilon$ is a very small positive contant). After the convergence of $C_{1}$ to $\ell_{i}, C_{2}$ is generated with its resultant $\kappa_{2}$ and $\delta_{2}$ on $G^{1}$ and $G^{2}$ to check $C_{2}\left(s_{0}\right)$ to be close to $\ell_{f}$ for $D_{2}^{e}$ in $\varepsilon$. In the non-converging case by clothoid parameter variation, arc segment is added as depicted in Fig. 3(b). In order for $C_{1}$ and $C_{2}$ to converge to each configuration, the circular arc segment $\widehat{p_{c} p_{c 1}}, \widehat{p_{c} p_{c 2}}$ are included to make $C_{r}$ by controlling its circular arc angle $\theta_{c 1}, \theta_{c 2}$ where $C_{r}=\rho_{c}^{\prime}\left(\theta_{c 1}+\theta_{c 2}\right)$ and $\theta_{c 2}$ is determined as the same way as $\theta_{c 1}$. Until the convergence of both segments to end configurations, the iterative variation on the parameters are performed for $\alpha_{1}, \delta_{1}, \theta_{c 1}$ and $\theta_{c 2}$.

The detailed procedure for finding clothoids parameters, taking into account the two cases given in Fig. 3, is described in Algorithm 1.

In Algorithm 1, initial sharpness $\alpha_{1 i}$ and deflection $\delta_{1 i}$ for $C_{1}$ are assumed given by designer before entering the loop.

It is noted that $\delta_{1 i}$ is set in the proposed work to $\frac{\left(\theta_{i}-\theta_{f}\right)}{2}$ from the fact of $\delta_{1}+\delta_{2}+\theta_{c}=\theta_{i}-\theta_{f}\left(G^{1}\right.$ continuity at $\left.p_{c}\right)$ with initially $\theta_{c}=0, \delta_{1}=\delta_{2}$. The $\alpha_{1 i}$ is set for $C_{1}\left(s_{0}\right)$ to be located between $\ell_{i}$ and $p_{c}$, i.e. if $C_{1}\left(s_{0}\right)$ resulting from $\delta_{1 i}$ and $\kappa_{c}=\frac{1}{\rho_{c}}$ is not located in $\ell_{i}$ and $p_{c}, \alpha_{1 i}$ is doubled until the convergence conditions (line 2, 4 in Algorithm 1) is satisfied. In line 2 to 3, the parameters are varied according to the property found as in Fig. 2 with (9). The convergence toward the solution is defined in line 4 where $D^{e}$, the distance between the composed curve $C_{1,2}$ and $\ell_{i, f}$ reach the threshold $\varepsilon$. The solution is always convergent as far as authors experience observed (there is not any encountered counter example 


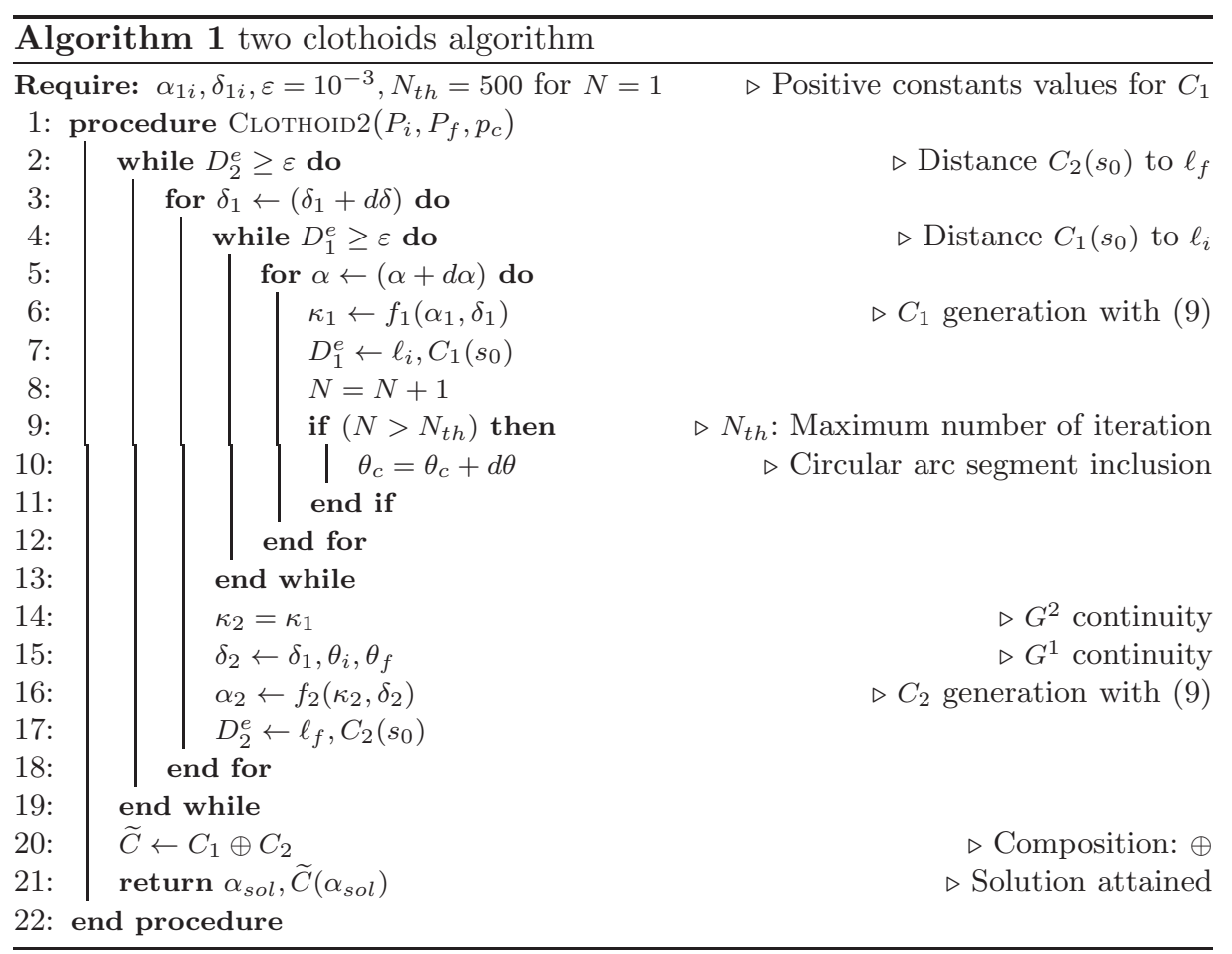

which do not lead to the desired configuration). Nevertheless, the convergence time depend obviously on the values of the set values of $d \alpha, d \delta$ and $d \theta$. This work is focusing its purpose on reconstructing a pre-obtained path and thus is not optimized for real-time planning. Its current convergence, while setting $d \alpha=10^{-2}, d \delta=10^{-1}$ and $d \theta=10^{-1}$ requires at least 20 to 50 iterations with more than a few seconds depending on the configurations. The efficiency linked to the rate of convergence remain an issue which will be addressed in near future.

\subsection{Global smooth path reconstruction}

To obtain autonomous navigation of vehicles, it is important to have smooth path, in the sens that frequent changes (or jerking) of the vehicle steering could increase undesirable noises and vibrations of the vehicle. These drawbacks, could cause the passenger discomfort but also some danger in high speed or at high curved road. In the proposed work, the smooth trajectory generated by continuous curvatures is applied to a test-bed of actual road environment. One of the performance to be evaluated is to smooth the real data acquired from the vehicle and then let it follow by a standard controller with comparative purpose.

To evaluate the proposed global smooth path reconstruction(cf. Algorithm 2 ), we will use in this set-up a raw date obtained by actual vehicle navigating in real environement. The points localization of the vehicle are recorded along its 
path. Algorithm 2 is used afterward for smoothing the path. The global path is divided to several local paths defined by two end configurations to be solved for each case using Algorithm 1. In this respect, the data is acquired from a DGPS which has positional accuracy less than $2 \mathrm{~cm}$ on the robotic vehicle of $1.3 \mathrm{~m}$ in width, $1.96 \mathrm{~m}$ in length and $1.5 \mathrm{~m}$ of the axis length in PAVIN test beds [14]. Each point data is sampled in $10 \mathrm{~Hz}$ and the vehicle tries to keep the velocity constant at $1 \mathrm{~m} / \mathrm{s}$.

Focusing on these raw data, the smoothed path generation is carried out by proposed solution where it should have continuous curvature while reconstructing as close as possible to the original experimental conditions. From the raw data acquired, it needs to set the boundary conditions for solving the clothoid segments problem as a local planner. For the general and automatic procedure that could be applied in the global framework, some pre-procedures are required to find the geometric boundary points in order to obtain the more appropriate clothoids for the corresponding conditions and constraints.

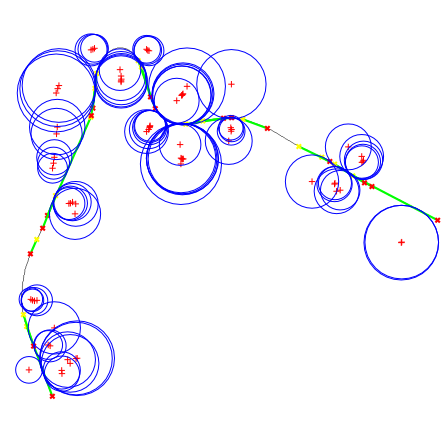

(a) Detection of linear/circular arc section

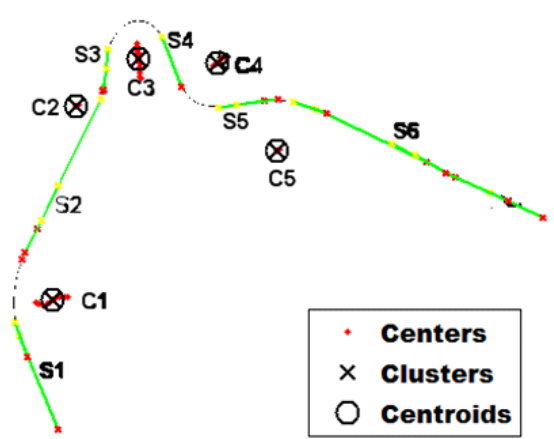

(b) Boundary conditions

Fig. 4. Procedure for extracting the boundary conditions

In the pre-procedures, the geometric data is mapped to the image plane and then the Hough Transform (HT) which is especially useful to extract the regional information for line (Hough Line Transform: HLT) or arc (Hough Circle Transform: HCT) [23] is performed on that image. The procedure is given in Algorithm 2.

In Algorithm 2, the overlapped lines are integrated into one segment and the circle are chosen to make boundary conditions to determine maximal curvature point $p_{c}$. For circular redundancy, firstly, the minimal radius is taken from the vehicle kinematic constraints i.e. $\kappa \in\left[\kappa_{\min }, \kappa_{\max }\right]$ and then the circles made from the straight regions (detected from HLT) are extracted out. Finally, the circular objects outside the turning curve are deleted.

The application of HT [23] for line and arc segments are performed as shown in Fig. 4(a). There are unnecessary information and noises in the arc results, 


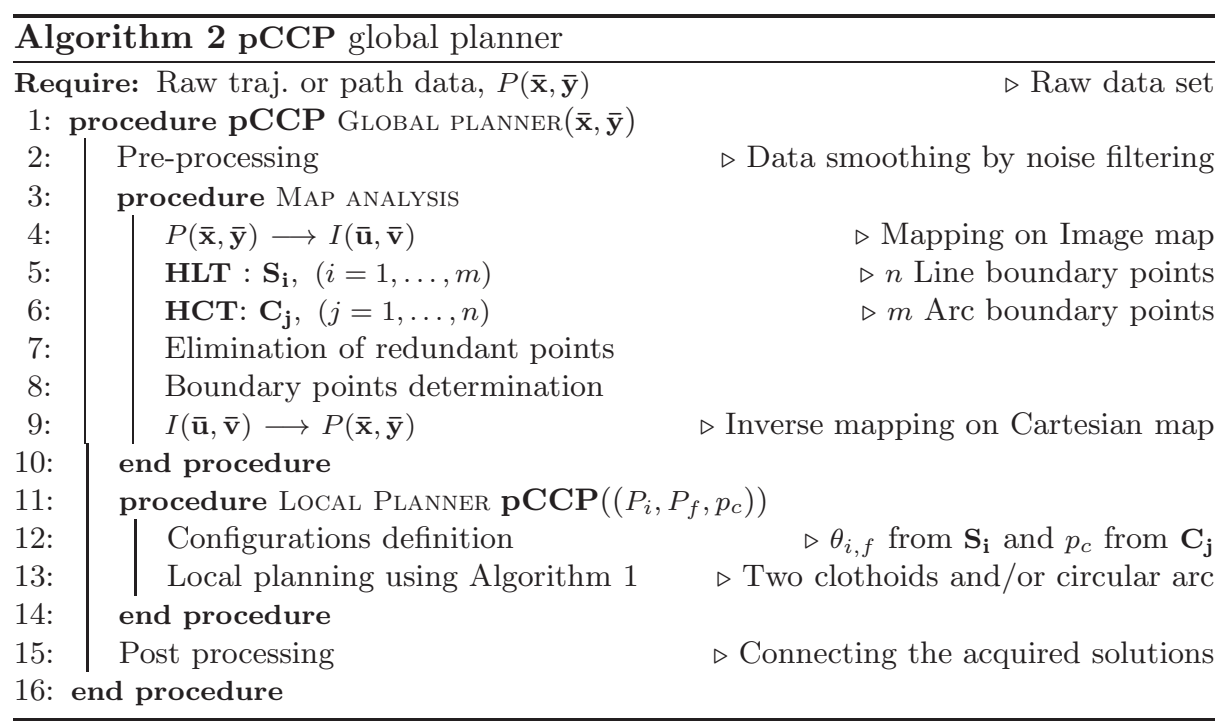

thus clustering by $k$-means nearest neighbor method are proceeded [24] and the cluster centers are sorted by minimal value of the distance summation as defined by,

$$
\min _{\hat{\mu}} \sum_{i \neq j}^{(i=p, j=q)}\left\|\sigma_{i}-\sigma_{j}\right\|,
$$

where $\sigma_{i}$ is the $i^{\text {th }}$ circle center in a cluster $\hat{\mu}$ and $\mathcal{L}^{2}$ (Euclidean) distance \|\| between the path and the circle center of minimal radius is selected as $p_{c}$ as shown in Fig. 3.

The integrated result in this procedure is shown in 4(b) where the line and clothoid/circular arc segments are noted by $\mathbf{S}_{\mathbf{i}}$ and $\mathbf{C}_{\mathbf{j}}$ for $i=1,2, \ldots, m$, $j=1,2, \ldots, n$ where $m, n$ are the number of the isolated segments respectively. From the results, isolation of straight and curved sections gives each problem for local planner pCCP, thus global continuous curvature trajectory is generated by connecting each solution result. Each solution has its own sharpness which is found from the geometric boundary configuration. Connected clothoids solution (cf. Algorithm 1 and 2) makes the continuous curvature diagram along the whole travel length. The diagram gives fully defined global reference trajectory in steering control with velocity information. In every segment of the diagram, the constant sharpness is found to meet the configuration where the curvature grows up to the maximal authorized curvature with the given boundary poses (initial and final configurations).

Fig. 5(a) shows the global pCCP results with comparison to the original path given by raw data. Fig. 5(b) displays the obtained curvature diagram along the whole travel length. Note that the curvature $\kappa(s)$ is expressed as $\kappa>0$ for its right turning. In Fig. 5(b), the curvature for raw data and its low pass filtered 


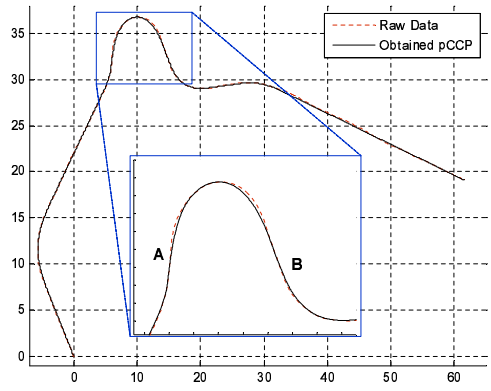

(a) Raw data and obtained path

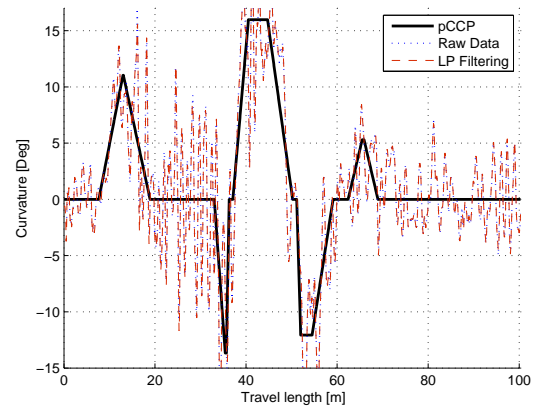

(b) Obtained curvature diagram

Fig. 5. Comparison of the path and the curvature diagram using global pCCP

data (using Butterworth filter for noise reduction) are plotted for comparison where its results are slightly more noisy than the obtained curvature by $\mathbf{~ C C P .}$.

The obtained curvature diagram (c.f. Fig. 5(b)) is continuous and smoothly drivable (inside each elementary local planning) over all the travel distance and it can be used thus efficiently to reconstruct a smooth reference path for vehicles.

\section{Simulation and Control Performance}

The obtained continuous curvature path was applied for autonomous vehicle navigation while using an appropriate Lyapunov based controller in the global framework described in Section 1. If the initial pose of the vehicle is exactly aligned to the beginning pose of the path, the vehicle follows the reference path without errors. However, any uncertainty in the actuation system, slippage on the road or time delays in controller could cause pose discrepancy to the expected values. Otherwise, additional sensors information to calibrate the pose are required and it also have to be stabilized by the used controller. The proposed algorithmic solution is tested using the Lyapunov based controller [18] for comparing its performance with the control using raw data (DGPS). The car-like vehicle starts from its initial pose with errors, $d_{e}=10^{-1} \mathrm{~m}$ and $\theta_{e}=10^{\circ}$ with constant velocity of $1 \mathrm{~m} / \mathrm{s}$.

Fig. 6 depicts the comparison results of two trajectories on the steering setpath and actual steering where the steering angle of the vehicle is controlled to follow the set-point. Fig. 6(b) shows explicitly the reduction of the fluctuation and smoother steering behaviors.

Fig. 7 displays two comparison results on the lateral pose and orientation error.With the above results including sharpness comparison, performance of the proposed path is compared to the result of the raw data. Performance comparisons are summarized in Table 1. 


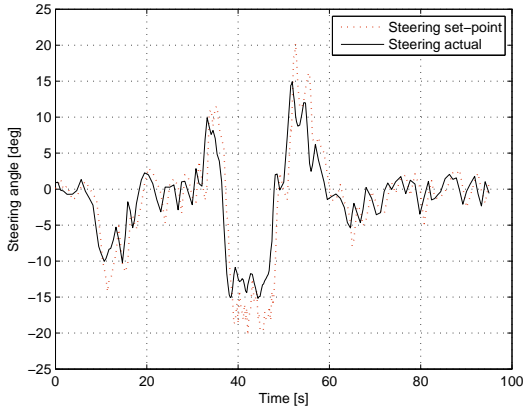

(a) Steering control for raw data

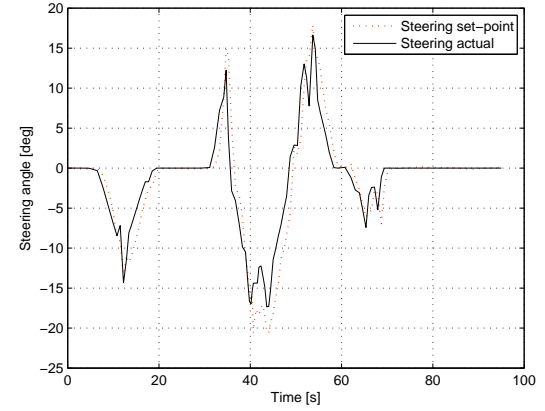

(b) Steering control for obtained path

Fig. 6. Comparison of steering control with/without pCCP

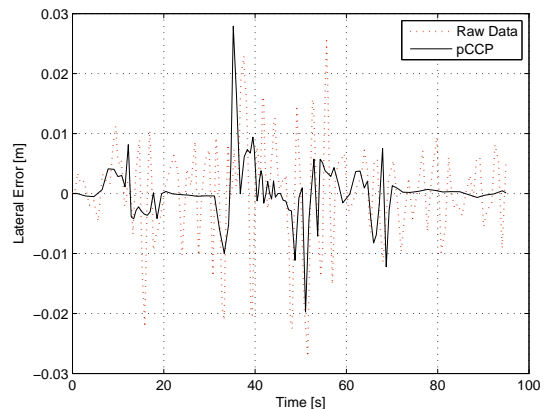

(a) Lateral pose error $\left(d_{e}\right)[\mathrm{m}]$

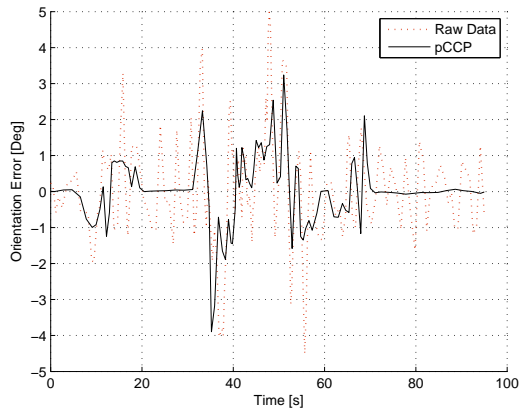

(b) Orientation error $\left(\theta_{e}\right)\left[^{\circ}\right]$

Fig. 7. Pose and orientation results

In Table 1, the performance are evaluated by quantitative as well as qualitative measures such that the peak values $(\max )$ evaluate the quality for each path and integrating or average $(a v g)$ values give the quantity level to analyze the performance in synthetical manner. The maximum and average lateral pose error are denoted by $p_{\max }^{e}$ and $p_{a v g}^{e}$ in $[m]$. It is also noted for orientation error $\theta_{\max }^{e}, \theta_{\text {avg }}^{e}$ in $\left[^{\circ}\right]$ and absolute sharpness by $\alpha_{\max }$ and $\alpha_{a v g}$ and for the maximal absolute steering $\kappa_{\max }$ in $\left[\frac{1}{m}\right]$ respectively. Note that average errors of position, orientation for pCCP are much less than the errors for raw data such as $54.4 \%$ and $42.6 \%$ respectively in its performance ratio. For the peak value on the errors of position and steering control, the performances of pCCP get worse than the raw data by $-2.6 \%$ and $-14.3 \%$ respectively. Analyzing the data results, the peak occurs around $35 \mathrm{sec}$. as given in Fig. 7(a) and marked as $\mathbf{A}$ in Fig. 5(a) (the region of $\mathbf{B}$ also makes the vehicle hard to follow in the same way). In that region, the vehicle of the original trajectory is assumed to make rapid turning 
Table 1. Performance Comparison

\begin{tabular}{|c|c|c|c|c|c|c|c|c|}
\hline Performance & $p_{\max }^{e}$ & $p_{\text {avg }}^{e}$ & $\theta_{\max }^{e}$ & $\theta_{\text {avg }}^{e}$ & $\left|\kappa_{\max }\right|$ & $\int|\kappa| d s$ & $|\alpha|_{\max }$ & $|\alpha|_{\text {avg }}$ \\
\hline Raw Data (a) & 0.0272 & 0.0068 & 0.0869 & 0.0202 & 15.1842 & 974.6058 & 23.4613 & 4.6522 \\
\hline pCCP (b) & 0.0279 & 0.0031 & 0.0680 & 0.0116 & 17.3489 & 574.8520 & 16.3169 & 2.4906 \\
\hline Ratio $\frac{a-b}{a}[\%]$ & -2.6 & 54.4 & 21.7 & 42.6 & -14.3 & 41.0 & 30.5 & 46.5 \\
\hline
\end{tabular}

of steering to follow the road profile where the curvature is also changed from positive to negative with high sharpness as shown in Fig. 5. Another possible reason is from the convergence threshold $D^{e}$ used in Algorithm 1 where the infenitesimal discontinuity occurs at the junction of the solution. This part of the problem will be subject to near future work.

The sharpness terms $\alpha_{\max }$ and $\alpha_{\text {avg }}$ get the advantage of $30.5 \%$ and $46.5 \%$ to the raw data and these validate that the steering behavior obtained using global $\mathbf{p C C P}$ is much smoother than the case using raw data.

The remarkable difference is also shown at the total steering work through the path $\int|\kappa| d s$. The steering work is reduced by $41.7 \%$ compared to the driving based on raw data. It shows that pCCP algorithm generates energysaving trajectory for the vehicle by smooth and continuous steering. Results given in Figs. 6 and 7, demonstrate that the smoothed path by clothoids segments gives an enhanced performance for the path following of the nonholonomic carlike vehicle. The reduced steering work and sharpness decrease permit to enhance passenger comfort by lessening the lateral acceleration and vehicle's jerk [2].

\section{Conclusion}

This paper proposed mainly an effective algorithm to generate a continuous curvature path using appropriate clothoid's parametric adjustment. The proposed algorithm, called Parametric Continuous Curvature Path ( $\mathbf{p C C P}$ ) could thus generates vehicles paths with smooth steering with lower lateral acceleration in energy-saving way. The effectiveness of using $\mathbf{~ P C C P}$ for a practical implementation was shown by accurate simulated results. The $\mathbf{p C C P}$ is afterward integrated in one proposed global planner which permits, among other, to transform recorded raw data corresponding to actual vehicle path, to one very close path w.r.t. the original one, but with smooth and continuous (in the sens of curvature) path. This kind of obtained smooth path is really desirable in order to enhance, for instance, the passenger comfort while using autonomous car-like vehicle. Indeed, in the showed simulation using standard controller, the vehicle could follow much better, the obtained path using global pCCP. In the future work, the performance of the proposed path generation algorithm will be used with an actual autonomous vehicle while considering uncertain errors, such as its motor actuation dynamics and/or the nonlinear tire contact with the road surface. 


\section{References}

[1] Montes, N., Mora, M.C., Tornero, J.: Trajectory generation based on rational Bezier curves as clothoids. IEEE Intel. Vehicles Symposium (2007) $505-510$

[2] Labakhua, L., Nunes, U., Rodrigues, R., Leite, F.S.: Smooth trajectory planning for fully automated passengers vehicles: Spline and Clothoid based methods and its simulation. In et al., A.C., ed.: Informatics in Control Automation and Robotics. Volume 15 of Lecture Notes Electrical Engineering. (2008) 159-182 [doi:10.1007/978-3-540-79142-3-14].

[3] Dubins, L.E.: On curves of minimal length with a constraint on average curvature, and with prescribed initial and terminal positions and tangents. American Journal of Mathematics 79 (1957) 497-516

[4] Reeds, J.A., Shepp, L.A.: Optimal paths for a car that goes both forwards and backwards. Pacific Journal of Mathematics 145(2) (1990) 367-393

[5] Fraichard, T., Scheuer, A.: From Reeds and Shepp's to continuous curvature paths. IEEE Trans. on Robotics 20 (2004) 1025-1035

[6] Girbes, V., Armesto, L., Tornero, J.: On generating continuous-curvature paths for line following problem with curvature and sharpness constraints. IEEE Int. Conf. on Robotics and Automation (2011) 6156-6161

[7] Choi, J.W., Curry, R.E., Elkaim, G.H.: Curvature-continuous trajectory generation with corridor constraint for autonomous ground vehicles. IEEE conf. on Decision and Control (2010) 7166-7171

[8] Villagra, J., Milantes, V., Perez, J., Godoy, J.: Smooth path and speed planning for an automated public transport vehicle. Robotics and Autonomous Systems 60 (2012) 252-265 [doi:10.1016/j.robot.2011.11.001].

[9] Brezak, M., Petrovic, I.: Real time approximation of clothoids with bounded error for path planning applications. IEEE Trans. on Robotics 30(2) (2014) 507-515

[10] Parlangeli, G., Indiveri, G.: Dubins inspired 2d smooth paths with bounded curvature and curvature derivative. Proc. of the 7th IFAC Symposium on Intelligent Autonomous Vehicles (2010) 252-257

[11] Walton, D.J., Meek, D.S.: A controlled clothoid spline. Computers and Graphics 29 (2005) 353-363

[12] Wilde, D.K.: Computing clothoid segments for trajectory generation. IEEE/RSJ Int. Conf. on Intel. Robots and Systems (Oct. 2009) 2440-2445

[13] Solea, R., Nunes, U.: Trajectory planning with velocity planner for fullyautomated passenger vehicle. Intel. Transportation Systems Conf. (Sep. 2006) 474-480

[14] Avanzini, P., Thuilot, B., Martinet, P.: Manual convoying of automated urban vehicles relying on monocular vision. Int. Vehicles Symposium (June. 2012) 19-24

[15] Khatib, O.: The potential field approach and operational space formulation in robot control. (1986) 367-377 
[16] Mouad, M., Adouane, L., Khadraoui, D., Martinet, P.: Mobile robot navigation and obstacles avoidance based on planning and re-planning algorithm. International IFAC Symposium on Robot Control (SYROCO'12) (5-7, September 2012)

[17] Meek, D.S., Walton, D.J.: An arc spline approximation to a clothoid. J. of Computation and Applied Mathematics 170 (2004) 59-77

[18] Canudas, C., Sicliano, B., Bastin, G.: Theory of Robot Control. Springer, ISBN 3-540-76054-7, Berlin Heidelberg (1996)

[19] DeLuca, A., Oriolo, G., Samson, C.: Feedback control of a nonholonoic carlike robot(Robot Motion Planning and Control). Springer-Verlag Berlin Heidelberg (1998)

[20] Soetanto, D., Lapierre, L., Pascoal, A.: Adaptive, non-singular pathfollowing control of dynamic wheeled robots. Proc. of IEEE Conf. on Decision and Control (1998) 1765-1770

[21] Samson, C.: Control of chained systems: application to path following and time-varying point stabilization of mobile robots. IEEE Trans. on Automatic Control 40(1) (1995) 64-77

[22] Piazzi, A., Bianco, C.G.: Quintic g2-splines for trajectory planning of autonomous vehicles. IEEE Intel. Vehicles Symposium (2000) 198-203

[23] Duda, R.O., Hart, P.E.: Use of the hough transformation to detect lines and curves in pictures. Graphics and Image Processing 15(1) (1972) 11-15

[24] Duda, R.O., Hart, P.E., Stork, D.G.: Pattern Classification. WileyInterscience (2001) 